\title{
Symptomatic Cerebral Edema Following Radiosurgery for AVMs
}

Can J Neurol Sci. 2013; 40: 765-766

This paper is a major original contribution to brain arteriovenous malformation (BAVM) management based on the extensive experience of Dr. Michael Schwartz and his colleagues. ${ }^{1}$ They reviewed the charts of 211 BAVM patients. Thirty-three patients were excluded because they were lost to follow-up or had less than 12 months follow-up. Ten patients had incomplete radiation dose data. Twenty patients (11.9\%) were determined to have either global or focal neurological deficits due to symptomatic brain edema following stereotactic radiosurgery (SRS) for BAVM. This compares with the rate of $8 \%$ reported in a multi-institutional study of complications of SRS. ${ }^{2}$ In addition to the term "symptomatic edema" the authors use symptomatic post-radiation T2 signal change, adverse treatment effects, adverse treatment outcomes, adverse radiation effects and clinically symptomatic postradiosurgery imaging changes to describe these non-hemorrhagic complications through their paper. The latter two terms can include abnormal contrast enhancement, which is not the subject of this paper. Symptomatic edema best describes what they are reporting.

The patients were treated with SRS using either a linear accelerator (LINAC) or Gamma Knife Surgery (GKS). Ninetysix patients were treated with Gamma Knife at the Toronto Western Hospital and 72 patients were treated with LINAC at the Sunnybrook Health Sciences Centre.

The authors studied the role of AVM angioarchitecture, which they have previously reported as an important factor in AVM obliteration by SRS. No other group has studied this factor in as much detail. The angioarchitecture of the AVMs was reviewed by neuroradiologists experienced in the interpretation of these lesions.

Symptoms related to complicating brain edema occurred at an average of 12.9 months following treatment. The BAVM predictors significantly associated with symptomatic edema were; non-hemorrhagic presentation, presence of venous rerouting, radiosurgery with Gamma Knife (compared to LINAC), and more than one draining vein.

The presence of a previous hemorrhage may be protective during SRS by causing a gliotic plane of tissue around the AVM, serving as a buffer zone.

The presence of venous rerouting, manifested by multiple draining veins, suggests to the authors that impaired venous outflow from the AVM leads to rerouting of arterialized blood into veins that drain the adjacent normal brain which leads to venous congestion which results in edema. One wonders whether changes in regional cerebral blood flow due to disturbed autoregulation may contribute to edema in a manner similar to that postulated for surgically treated BAVMs by Spetzler and colleagues. ${ }^{3}$ Brain arteriovenous malformation treated by SRS are obliterated at varying rates and it is possible that there is an element of "normal perfusion pressure breakthrough" as the AVM is in the final stages of obliteration.
The difference between the results from GKS and LINAC treatment deserves comment. The radiation dose during GKS varied from 25 Gy to the $50 \%$ isodose contour for AVMs with less than $4 \mathrm{cc}$ volume to $20 \mathrm{~Gy}$ to the $50 \%$ isodose contour for those measuring greater than $4 \mathrm{cc}$ in volume. The maximum dose with GKS was $50 \mathrm{~Gy}$. In the LINAC group the radiation dose to AVMs in eloquent brain tissues was 15 Gy to the $67 \%$ isodose contour and for AVMs in non-eloquent brain the dose was 20 Gy to the $90 \%$ isodose contour. The maximum dose with LINAC was $25 \mathrm{~Gy}$. Symptomatic edema occurred in $17.7 \%$ of GKS patients and $4 \%$ of LINAC patients. The difference in outcomes is due to the significant difference in the maximum dose of radiation prescribed, rather than the technology employed to deliver the SRS. Is it possible that the tight conformity (resulting from the use of more isocenters) of the GKS treatments led the operators to prescribe higher doses than may have been used when the conformity of the treatment plan was not as tight?

Age, AVM location, and AVM volume did not reach statistical significance as predictors of symptomatic edema in this report. However, the BAVM literature has documented that complications of SRS are related to radiation dose and AVM volume. ${ }^{4}$ The volume of tissue receiving a dose of $12 \mathrm{~Gy}$ or more has been shown to correlate with the risk of imaging changes following SRS within a range of doses. The location of the AVM has been described as an important factor by Flickinger et al. ${ }^{5}$ Pollock and Flickinger developed a grading system to predict which patients may undergo complete AVM obliteration following SRS without developing new neurological deficit which is dependent on AVM location, AVM volume, and patient age. ${ }^{6}$

The authors have made a very important contribution to the management of the patients who are treated with SRS for BAVMs. It remains to be shown how knowledge of the angioarchitectural characteristics of the BAVM may be used to reduce the complications of treatment. The previously documented factors of AVM volume, patient age, and AVM location should continue to be considered when planning treatment for these complex lesions.

Specialists in SRS will be interested to know the details of the authors' treatment regime for symptomatic edema.

The authors point out the limitations of their study which has a relatively small number of patients, is retrospective, and is characterized by heterogeneity of the patient population for preSRS treatment and treatment modality. The patients were treated at two different hospitals, with a longer experience with LINAC than GKS.

\author{
Michael West \\ University of Manitoba, Winnipeg, Manitoba, Canada \\ Email:mwest@hsc.mb.ca
}




\section{REFERENCES}

1. Machnowska M, Taeshineetanakul P, Geibprasert S, et al. Factors determining the clinical complications of radiosurgery for AVM. Can J Neuro Sci. 2013;40(6):807-13.

2. Flickinger JC, Kondziolka D, Lunsford LD, et al. A multiinstitutional analysis of complication outcomes after arteriovenous malformation radiosurgery. Int J Radiat Oncol Biol Phys. 1999;44:67-74.

3. Spetzler RF, Wilson CB, Weinstein $\mathrm{P}$, et al. Normal perfusion pressure breakthrough theory. Clin Neurosurg. 1978;25:651-72.
4. Flickinger JC, Kondziolka D, Kalend AM, et al. Radiosurgeryrelated imaging changes in surrounding brain: multivariate analysis and model evaluation. Radiosurgery. 1996;1:229-36.

5. Flickinger JC, Kondziolka D, Maitz AH, et al. Analysis of neurological sequelae from radiosurgery of arteriovenous malformations: how location affects outcome. Int J Radiat Oncol Biol Phys. 1998;40(2):273-8.

6. Pollock BE, Flickinger JC. Modification of the radiosurgery-based arteriovenous malformation grading system. Neurosurgery. 2008;63:239-43. 